Pacific Journal of Mathematics

ON THE RENEWAL FUNCTION WHEN SOME OF THE MEAN 


\section{ON THE RENEWAL FUNCTION WHEN SOME OF THE MEAN RENEWAL LIFETIMES ARE INFINITE}

\section{MaKoto MaEJIMA}

Let $\left\{X_{i}, i=1,2, \cdots\right\}$ be a sequence of independent and nonnegative random variables with the distribution function $F_{i}(x)$. Some of $\int_{0}^{\infty} x d F_{i}(x)$ may be infinite. Let $H(t)$ be the renewal function. The main object of this note is to show that in order to have the asymptotic relation $H(t) / t \sim 1 / L(t)$ as $t \rightarrow \infty$, it is necessary and sufficient that $\mu(t) \sim L(t)$ as $t \rightarrow \infty$, where $L(t)$ is a function of slow growth and $\mu(t)=$ $\lim _{n \rightarrow \infty}(1 / n) \sum_{i=1}^{n} \mu_{i}(t), \mu_{i}(t)$ being $\int_{0}^{t}\left[1-F_{i}(x)\right] d x$, is supposed to exist uniformly in $t$.

Let $H(t)$ be the renewal function for a renewal process, that is, a sequence $\left\{X_{i}, i=1,2, \cdots\right\}$ of nonnegative, independent and identically distributed random variables. Namely $H(t)=E N(t)=E\left[\sup \left\{n ; S_{n} \leqq t\right\}\right]$, where $S_{n}=\sum_{i=1}^{n} X_{i}$. Smith [3] has studied the limiting behaviors of $H(t) / t$ for the case in which $E X_{i}=\infty$.

We now consider an extended renewal process in which $X_{i}$, $i=1,2, \cdots$ may not be identically distributed. We also in this case use the similar notations $S_{n}$ and $N(t)$, and we may also define $H(t)$ in the similar manner under the condition that $S_{n}$ has no finite limit point. The main object of this note is to give a generalization of a result of Smith to our extended case.

2. Some lemmas. We begin with some lemmas for an extended renewal process with the finite mean lifetimes.

Let $\left\{X_{i}, i=1,2, \cdots\right\}$ be a sequence of independent and nonnegative random variables with $0<E X_{i}=\mu_{i}<\infty$ and let $F_{i}(x)$ be the distribution function of $X_{i}$.

Lemma 1. Suppose that

$$
\mu=\lim _{n \rightarrow \infty} \frac{1}{n} \sum_{i=1}^{n} \mu_{i}>0
$$

exists and that

$$
\lim _{A \rightarrow \infty} \int_{A}^{\infty} x d F_{i}(x)=0
$$

holds uniformly with respect to $i$. Then we have $E N^{\alpha}(t)<\infty$ for each $t>0$, for $\alpha=1,2, \cdots$. 
This lemma was first proved by Kawata [2] for $\alpha=1$, and Hatori [1] showed it for any positive integer $\alpha$.

LEMma 2. Suppose that $E N(t)$ and $E N^{2}(t)$ are finite and that (2.1) is true. Then we have for every $t$

$$
E S_{N(t)+1}=\mu(H(t)+1)+\sum_{n=1}^{\infty} n \varepsilon_{n} \operatorname{Pr}\{N(t)+1=n\},
$$

where $\varepsilon_{n}$ is defined by

$$
\varepsilon_{n}=\frac{1}{n} \sum_{i=1}^{n} \mu_{i}-\mu
$$

which converges to zero as $n \rightarrow \infty$.

Proof. Letting

$$
\begin{aligned}
Z_{n} & =1, \text { if } n \leqq N(t)+1 \\
& =0, \text { otherwise, }
\end{aligned}
$$

we have

$$
E S_{N(t)+1}=E \sum_{n=1}^{N(t)+1} X_{n}=E \sum_{n=1}^{\infty} X_{n} Z_{n}
$$

Since

$$
\begin{aligned}
\left\{Z_{n}=0\right\} & =\{N(t)+1<n\}=\bigcup_{k=1}^{n-1}\{N(t)+1=k\} \\
& =\left\{X_{1}>t\right\} \cup \bigcup_{k=2}^{n-1}\left\{\left(X_{1}+\cdots+X_{k-1} \leqq t\right) \cap\left(X_{1}+\cdots+X_{k}>t\right)\right\},
\end{aligned}
$$

$Z_{n}$ is independent of $X_{n}$. Thus, noticing the nonnegativeness of $X_{n}$, we see that $(2.2)$ is

$$
\sum_{n=1}^{\infty} E X_{n} Z_{n}=\sum_{n=1}^{\infty} E X_{n} E Z_{n}=\sum_{n=1}^{\infty} \mu_{n} \operatorname{Pr}\{N(t)+1 \geqq n\},
$$

which turns out to be

$$
\begin{aligned}
E S_{N(t)+1} & =\sum_{n=1}^{\infty}\left(\mu+n \varepsilon_{n}-(n-1) \varepsilon_{n-1}\right) \operatorname{Pr}\{N(t)+1 \geqq n\} \\
& =\mu(H(t)+1)+\sum_{n=1}^{\infty}\left(n \varepsilon_{n}-(n-1) \varepsilon_{n-1}\right) \operatorname{Pr}\{N(t)+1 \geqq n\} .
\end{aligned}
$$

Since

$$
\sum_{n=1}^{\infty}\left|n \varepsilon_{n} \operatorname{Pr}\{N(t)+1 \geqq n\}\right| \leqq \sup _{n}\left|\varepsilon_{n}\right|\left(E N^{2}(t)+2\right)<\infty
$$

by the finiteness of $E N^{2}(t)$, we may rewrite 


$$
\sum_{n=1}^{\infty}\left(n \varepsilon_{n}-(n-1) \varepsilon_{n-1}\right) \operatorname{Pr}\{N(t)+1 \geqq n\}=\sum_{n=1}^{\infty} n \varepsilon_{n} \operatorname{Pr}\{N(t)+1=n\},
$$

so that

$$
E S_{N(t)+1}=\mu(H(t)+1)+\sum_{n=1}^{\infty} n \varepsilon_{n} \operatorname{Pr}\{N(t)+1=n\},
$$

which is the conclusion.

3. A theorem. We return to the case where $X_{i}$ may have the infinite mean renewal lifetimes. Let $L(t)$ be a function of slow growth, that is, for every fixed $c>0, L(c t) / L(t) \rightarrow 1$ as $t \rightarrow \infty$. We shall show the following theorem which is an extension of a result due to Smith ([3], Theorem 1, (i), $\nu=1$ ) to the case of nonidentically distributed random variables.

Theorem. Let $\left\{X_{i}, i=1,2, \cdots\right\}$ be a sequence of independent and nonnegative random variables with the distribution function $F_{i}(x)$. Suppose that

$$
\mu(t)=\lim _{n \rightarrow \infty} \frac{1}{n} \sum_{i=1}^{n} \mu_{i}(t)>0
$$

exists uniformly in $0<t<\infty$, where

$$
\mu_{i}(t)=\int_{0}^{t}\left[1-F_{i}(x)\right] d x .
$$

Then the necessary and sufficient condition for the validity of the asymptotic relation

$$
\frac{H(t)}{t} \sim \frac{1}{L(t)}, \text { as } t \rightarrow \infty,
$$

where $L(t)$ is a function of slow growth, is that

$$
\mu(t) \sim L(t), \text { as } t \rightarrow \infty .
$$

Before proving the theorem we shall show some lemmas.

We now define a new renewal process $\left\{X_{i}^{*}\right\}$ for a fixed positive number $t^{*}$ by putting

$$
\begin{aligned}
X_{i}^{*} & =X_{i}, \quad \text { if } X_{i} \leqq t^{*}, \\
& =t^{*}, \quad \text { otherwise } .
\end{aligned}
$$

We note that $E X_{i}^{*}=\mu_{i}\left(t^{*}\right)$ is finite. For the new variables $X_{i}^{*}$, we define $S_{n}^{*}, N^{*}(t)$ and $H^{*}(t)$ in obvious ways. Then we may easily 
verify the conditions of Lemma 1 for a fixed $t^{*}$ and the following lemma is immediate.

Lemma 3. Suppose that (3.1) exists for $t^{*}$. Then $E\left\{N^{*}(t)\right\}^{\alpha}<\infty$ for $\alpha=1,2, \cdots$.

The next two lemmas play essential roles in the proof of Theorem.

Lemma 4. Suppose that (3.1) exists uniformly in $t$. Then we have

$$
\liminf _{t \rightarrow \infty} \frac{H(t) \mu(t)}{t} \geqq 1
$$

Proof. We consider $X_{i}^{*}$ defined above. Since $E N^{*}(t)$ and $E\left\{N^{*}(t)\right\}^{2}$ are finite by Lemma 3 , we have that for all $t$,

$$
t<\mu\left(t^{*}\right)\left(H^{*}(t)+1\right)+\sum_{n=1}^{\infty} n \varepsilon_{n}\left(t^{*}\right) \operatorname{Pr}\left\{N^{*}(t)+1=n\right\}
$$

by Lemma 2 and noting $t<S_{N(t)+1}$, where $\varepsilon_{n}\left(t^{*}\right)$ is defined by

$$
\frac{1}{n} \sum_{i=1}^{n} \mu_{i}\left(t^{*}\right)=\mu\left(t^{*}\right)+\varepsilon_{n}\left(t^{*}\right) \text {. }
$$

Now (3.4) holds for $t=t^{*}$, in particular. Thus we have

$$
t^{*}<\mu\left(t^{*}\right)\left(H^{*}\left(t^{*}\right)+1\right)+\sum_{n=1}^{\infty} n \varepsilon_{n}\left(t^{*}\right) \operatorname{Pr}\left\{N^{*}\left(t^{*}\right)+1=n\right\} .
$$

Next, we estimate of the order of $\varepsilon_{n}(t)$ as $t \rightarrow \infty$. Since the function $1-F_{i}(x)$ decreases to zero as $x \rightarrow \infty$, so does $\mu_{i}(t) / t$ as $t \rightarrow \infty$. In view of the assumption that (3.1) exists uniformly in $t$, it follows that, for any $\varepsilon>0$, there exists a constant $N$ independent of $t$ such that

$$
\left|\mu(t)-\frac{1}{n} \sum_{i=1}^{n} \mu_{i}(t)\right|<\varepsilon, \text { for } n \geqq N .
$$

Then we have

$$
\begin{aligned}
\frac{1}{t}|\mu(t)| & \leqq \frac{1}{t}\left|\mu(t)-\frac{1}{N} \sum_{i=1}^{N} \mu_{i}(t)\right|+\frac{1}{t} \cdot \frac{1}{N} \sum_{i=1}^{N}\left|\mu_{i}(t)\right| \\
& <\frac{\varepsilon}{t}+\varepsilon<2 \varepsilon
\end{aligned}
$$

for sufficiently large $t$, taking into account the fact that $\mu_{i}(t) / t \rightarrow 0$ as $t \rightarrow \infty$. Thus, we have for sufficiently large $t$ 


$$
\frac{1}{t}\left|\varepsilon_{n}(t)\right|=\frac{1}{t}\left|\mu(t)-\frac{1}{n} \sum_{i=1}^{n} \mu_{i}(t)\right|<\frac{\varepsilon}{N^{2}},
$$

for the fixed $N$ and for all $n \leqq N$. Therefore we have, for large $t^{*}$, from (3.6) and (3.7)

$$
\begin{aligned}
& \sum_{n=1}^{\infty} n \varepsilon_{n}\left(t^{*}\right) \operatorname{Pr}\left\{N^{*}\left(t^{*}\right)+1=n\right\} \\
< & \frac{\varepsilon}{N^{2}} t^{*} \sum_{n=1}^{N} n \operatorname{Pr}\left\{N^{*}\left(t^{*}\right)+1=n\right\}+\varepsilon \sum_{n=N+1}^{\infty} n \operatorname{Pr}\left\{N^{*}\left(t^{*}\right)+1=n\right\} \\
< & \varepsilon\left(t^{*}+H^{*}\left(t^{*}\right)+1\right) .
\end{aligned}
$$

Now we shall show that

$$
\limsup _{t^{*} \rightarrow \infty} \frac{H^{*}\left(t^{*}\right)}{t^{*}}<\infty .
$$

In order to show this, we define new truncated random variables $X_{i, A}$ for some constant $A$ by putting

$$
\begin{aligned}
X_{i, A} & =X_{i}, \quad \text { if } X_{i} \leqq A, \\
& =A, \quad \text { otherwise } .
\end{aligned}
$$

Clearly $E X_{i, A}=\mu_{i}(A)$ is finite and by the elementary renewal theorem for an extended renewal process, we have that, if $H_{A}(t)$ is the renewal function associated with $\left\{X_{i, A}\right\}$, then

$$
\lim _{t \rightarrow \infty} \frac{H_{A}(t)}{t}=\frac{1}{\mu(A)} .
$$

(For details, see Kawata [2].) (3.9) follows from the remark that $H^{*}\left(t^{*}\right) \leqq H_{A}\left(t^{*}\right)$ for $t^{*} \geqq A$. Since $\varepsilon$ is arbitrary in (3.8), we have from (3.8)

$$
\lim _{t^{*} \rightarrow \infty} \frac{1}{t^{*}}\left|\sum_{n=1}^{\infty} n \varepsilon_{n}\left(t^{*}\right) \operatorname{Pr}\left\{N^{*}\left(t^{*}\right)+1=n\right\}\right|=0 .
$$

Therefore, from (3.5)

$$
\liminf _{t^{*} \rightarrow \infty} \frac{1}{t^{*}} \mu\left(t^{*}\right)\left(H^{*}\left(t^{*}\right)+1\right) \geqq 1 .
$$

On the other hand, we have

$$
\operatorname{Pr}\left\{S_{n}^{*} \leqq t^{*}\right\}=\operatorname{Pr}\left\{S_{n} \leqq t^{*}\right\},
$$

for $n=2,3, \cdots$, and

$$
\operatorname{Pr}\left\{S_{1}^{*} \leqq t^{*}\right\}=1
$$

Thus 


$$
H^{*}\left(t^{*}\right)=H\left(t^{*}\right)+\operatorname{Pr}\left\{X_{1}>t^{*}\right\}
$$

and so

$$
\liminf _{t \rightarrow \infty} \frac{1}{t} \mu(t)\left(H(t)+\operatorname{Pr}\left\{X_{1}>t\right\}+1\right) \geqq 1 .
$$

Noticing that $\mu(t) / t \rightarrow 0$ as $t \rightarrow \infty$, we have the conclusion of the lemma.

LEMMA 5. Under the same conditions as in Lemma 4, we have for arbitrary $\delta>0$

$$
\limsup _{t \rightarrow \infty} \frac{H(t) \mu(\delta t)}{t} \leqq 1+\delta
$$

Proof. Take $\delta>0$ arbitrarily and let $\hat{X}_{n}$ represent new variables truncated according to the rule

$$
\begin{aligned}
\hat{X}_{n} & =X_{n}, \quad \text { if } X_{n} \leqq \delta t^{*}, \\
& =\delta t^{*},
\end{aligned}
$$

It is clear that $E \hat{X}_{n}=\mu_{n}\left(\delta t^{*}\right)<\infty$. Then, noting that $t \geqq S_{N(t)+1}-$ $X_{N(t)+1}$, we have, by Lemma 2,

$$
\begin{aligned}
t & \geqq \mu\left(\delta t^{*}\right)(\hat{H}(t)+1)+\sum_{n=1}^{\infty} n \varepsilon_{n}\left(\delta t^{*}\right) \operatorname{Pr}\{\hat{N}(t)+1=n\}-E \hat{X}_{\hat{N}(t)+1} \\
& \geqq \mu\left(\delta t^{*}\right)(\hat{H}(t)+1)+\sum_{n=1}^{\infty} n \varepsilon_{n}\left(\delta t^{*}\right) \operatorname{Pr}\{\hat{N}(t)+1=n\}-\delta t^{*},
\end{aligned}
$$

where $\hat{N}(t)$ and $\hat{H}(t)$ are defined in the renewal process associated with the new truncated variables $\left\{\hat{X}_{n}\right\}$. Since (3.11) holds for $t=t^{*}$, in particular, we have

$$
(1+\delta) t^{*} \geqq \mu\left(\delta t^{*}\right)\left(\hat{H}\left(t^{*}\right)+1\right)+\sum_{n=1}^{\infty} n \varepsilon_{n}\left(\delta t^{*}\right) \operatorname{Pr}\left\{\hat{N}\left(t^{*}\right)+1=n\right\} .
$$

The same arguments as in the proof of Lemma 4 yield that

$$
\lim _{t^{*} \rightarrow \infty} \frac{1}{t^{*}}\left|\sum_{n=1}^{\infty} n \varepsilon_{n}\left(\delta t^{*}\right) \operatorname{Pr}\left\{\hat{N}\left(t^{*}\right)+1=n\right\}\right|=0
$$

for the fixed $\delta>0$. Noting that

$$
\hat{H}\left(t^{*}\right) \geqq H\left(t^{*}\right),
$$

we have the required result.

We now turn to the proof of the theorem.

Proof of Theorem. We first assume that 


$$
\frac{H(t)}{t} \sim \frac{1}{L(t)}, \quad \text { as } \quad t \rightarrow \infty
$$

By Lemma 4 we have

$$
\liminf _{t \rightarrow \infty} \frac{\mu(t)}{L(t)} \geqq 1
$$

and by Lemma 5 , for any $\delta>0$,

$$
\limsup _{t \rightarrow \infty} \frac{\mu(\delta t)}{L(t)} \leqq 1+\delta
$$

Writing $\delta t$ for $t$, and using the fact that $L(t / \delta) \sim L(t)$ as $t \rightarrow \infty$, we have

$$
\limsup _{t \rightarrow \infty} \frac{\mu(t)}{L(t)} \leqq 1+\delta
$$

Since $\delta$ can be arbitrarily small, we, taking into account (3.13), conclude the necessity part.

Furthermore, in view of the assumption $\mu(t)$ is a function of slow growth, it follows by Lemma 5 that

$$
\limsup _{t \rightarrow \infty} \frac{H(t) \mu(t)}{t}<1+\delta \text {. }
$$

Since $\delta$ is arbitrary, Lemma 4 gives the sufficiency part.

When $\lim _{t \rightarrow \infty} \mu(t)=\infty$, we can relax slightly the condition of the uniform existence of $\mu(t)$ in the following way.

Corollary. Suppose that

$$
\mu(t)=\lim _{n \rightarrow \infty} \frac{1}{n} \sum_{i=1}^{n} \mu_{i}(t)>0
$$

exists for all $t$, (not necessarily uniformly), and that there exists a constant $K$, independent of $t$, such that

$$
\left|\mu(t)-\frac{1}{n} \sum_{i=1}^{n} \mu_{i}(t)\right|=\left|\varepsilon_{n}(t)\right|<K
$$

for $n \geqq N, N$ being some finite positive integer. If $\lim _{t \rightarrow \infty} \mu(t)=\infty$, then the necessary and sufficient condition for the validity of the asymptotic relation (3.2) is (3.3).

Proof. In the proof of theorem, the condition relaxed has been used only in order to show (3.10) and (3.12). Thus, it suffices to show that (3.10) holds under the conditions of this corollary. 
Now, we have

$$
\begin{aligned}
\frac{1}{t}|\mu(t)| & \leqq \frac{1}{t}\left|\mu(t)-\frac{1}{N} \sum_{i=1}^{N} \mu_{i}(t)\right|+\frac{1}{t} \cdot \frac{1}{N} \sum_{i=1}^{N}\left|\mu_{i}(t)\right| \\
& <\frac{K}{t}+\frac{1}{N} \sum_{i=1}^{N}\left|\frac{\mu_{i}(t)}{t}\right|,
\end{aligned}
$$

and so $|\mu(t)| / t$ can be arbitrarily small for the sufficiently large $t$. Thus, $\varepsilon_{n}(t)=o(t)$ for all $n \leqq N$. Therefore, we have

$$
\begin{aligned}
& \left|\sum_{n=1}^{\infty} n \varepsilon_{n}\left(t^{*}\right) \operatorname{Pr}\left\{N^{*}\left(t^{*}\right)+1=n\right\}\right| \\
< & o\left(t^{*}\right) \sum_{n=1}^{N} n \operatorname{Pr}\left\{N^{*}\left(t^{*}\right)+1=n\right\}+K \sum_{n=N+1}^{\infty} n \operatorname{Pr}\left\{N^{*}\left(t^{*}\right)+1=n\right\} \\
< & o\left(t^{*}\right) N^{2}+K\left(H^{*}\left(t^{*}\right)+1\right) .
\end{aligned}
$$

Now we shall show under the condition that $\mu(t) \rightarrow \infty$, that

$$
\lim _{t^{*} \rightarrow \infty} \frac{H^{*}\left(t^{*}\right)}{t^{*}}=0
$$

As in the proof of the previous theorem, we have

$$
\limsup _{t^{*} \rightarrow \infty} \frac{H^{*}\left(t^{*}\right)}{t^{*}} \leqq \lim _{t^{*} \rightarrow \infty} \frac{H_{A}\left(t^{*}\right)}{t^{*}}=\frac{1}{\mu(A)} .
$$

Since $A$ is arbitrary, this shows that

$$
\lim _{t^{*} \rightarrow \infty} \frac{H^{*}\left(t^{*}\right)}{t^{*}}=0
$$

and (3.10) holds.

The author wishes to express his sincere appreciation to Professor Tatsuo Kawata of Keio University for continuing guidances and encouragements.

\section{REFERENCES}

1. H. Hatori, A note on a renewal theorem, Kodai Math. Sem. Rep., 12 (1960), 28-37.

2. T. Kawata, A renewal theorem, J. Math. Soc. Japan, 8 (1956), 118-126.

3. W. L. Smith, $A$ note on the renewal function when the mean renewal lifetime is infinite, J. Roy. Stat. Soc., B23 (1961), 230-237.

Received March 3, 1973.

KEIO UNIVERSITY 


\section{PACIFIC JOURNAL OF MATHEMATICS}

\section{EDITORS}

RICHARD ARENS (Managing Editor) University of California

Los Angeles, California 90024

R. A. Beaumon'T

University of Washington Seattle, Washington 98105
J. Dugundj1*

Department of Mathematics University of Southern California Los Angeles, California 90007

D. Gilbarg and J. Milgram Stanford University Stanford, California 94305

\section{ASSOCIATE EDITORS}

E. F. BECKENBACH

B. H. NeUMANN

F. WOLF

K. YOSHIDA

\section{SUPPORTING INSTITUTIONS}

UNIVERSITY OF BRITISH COLUMBIA CALIFORNIA INSTITUTE OF TECHNOLOGY UNIVERSITY OF CALIFORNIA MONTANA STATE UNIVERSITY UNIVERSITY OF NEVADA NEW MEXICO STATE UNIVERSITY OREGON STATE UNIVERSITY UNIVERSITY OF OREGON OSAKA UNIVERSITY

\section{UNIVERSITY OF SOUTHERN CALIFORNIA STANFORD UNIVERSITY UNIVERSITY OF TOKYO UNIVERSITY OF UTAH WASHINGTON STATE UNIVERSITY UNIVERSITY OF WASHINGTON AMERICAN MATHEMATICAL SOCIETY NAVAL WEAPONS CENTER}

* C. R. DePrima California Institute of Technology, Pasadena, CA 91109, will replace J. Dugundji until August 1974. 


\section{Pacific Journal of Mathematics}

\section{Vol. 49, No. $1 \quad$ May, 1973}

A. Bigard, Free lattice-ordered modules ...........................

Richard Bolstein and Warren R. Wogen, Subnormal operators in strictly cyclic

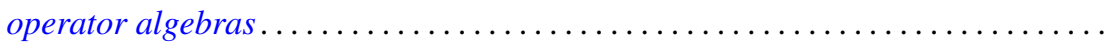

Herbert Busemann and Donald E. Glassco, II, Irreducible sums of simple

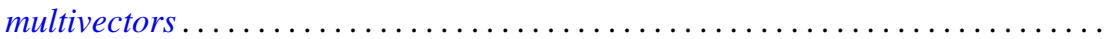

W. Wistar (William) Comfort and Victor Harold Saks, Countably compact groups

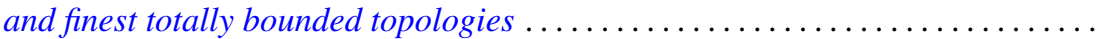

Mary Rodriguez Embry, Maximal invariant subspaces of strictly cyclic operator

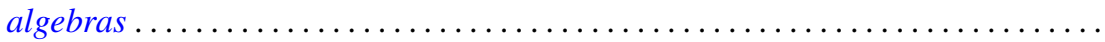

Ralph S. Freese and James Bryant Nation, Congruence lattices of semilattices......

Ervin Fried and George Grätzer, A nonassociative extension of the class of

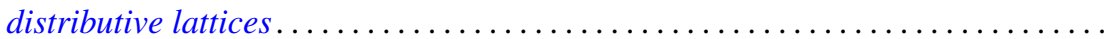

John R. Giles and Donald Otto Koehler, On numerical ranges of elements of locally

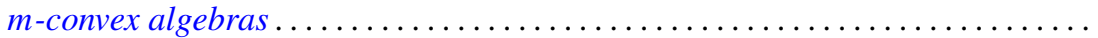

David A. Hill, On dominant and codominant dimension of $\mathrm{QF}-3$ rings ........ John Sollion Hsia and Robert Paul Johnson, Round and Pfister forms over $R(t) \ldots$ I. Martin (Irving) Isaacs, Equally partitioned groups . . . . . . . . . . . . . .

Athanassios G. Kartsatos and Edward Barry Saff, Hyperpolynomial approximation of solutions of nonlinear integro-differential equations.

Shin'ichi Kinoshita, On elementary ideals of $\theta$-curves in the 3-sphere and 2-links in the 4-sphere

Ronald Brian Kirk, Convergence of Baire measures

R. J. Knill, The Seifert and Van Kampen theorem via regular covering spaces ..

Amos A. Kovacs, Homomorphisms of matrix rings into matrix rings ..

Young K. Kwon, HD-minimal but no $H D$-minimal ..........

Makoto Maejima, On the renewal function when some of the mean renewal lifetimes are infinite

Juan José Martínez, Cohomological dimension of discrete modules over profinite groups.

W. K. Nicholson, Semiperfect rings with abelian group of units

Louis Jackson Ratliff, Jr., Three theorems on imbedded prime divisors of principal ideals.

Billy E. Rhoades and Albert Wilansky, Some commutants in $B(c)$ which are almost matrices

John Philip Riley Jr., Cross-sections of decompositions . . .

Keith Duncan Stroyan, A characterization of the Mackey uniformity $m\left(L^{\infty}, L^{1}\right)$ for

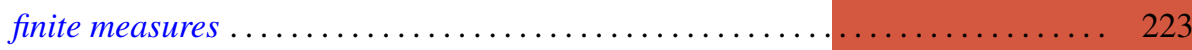

Edward G. Thurber, The Scholz-Brauer problem on addition chains . . . . . . . . . 229

Joze Vrabec, Submanifolds of acyclic 3-manifolds ............

Philip William Walker, Adjoint boundary value problems for compactified singular

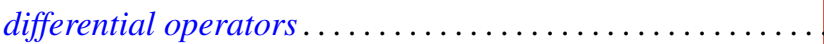

